phys. stat. sol. (b) 155, 349 (1989)

Subject classification: 61.30 and 62.10

Institut für Theoretische und Angewandte Physik der Universität Stuttgart ${ }^{1}$ )

\title{
Temperature Dependence of the Elastic Constants for Biaxial Nematic Liquid Crystals
}

By

\section{Monselesan and H.-R. Trebin}

The elastic constants $K_{i j}$ of the Frank-Oseen energy density for uniaxial nematic liquid crystals depend on the Maier-Saupe order parameter $S$ and hence on temperature. Longa et al. recently used an extended Landau-Ginzburg-de Gennes theory to expand the functions $K_{i j}(S)$ up to fourth order in $S$. Here, a similar procedure is applied for the elastic energy density of biaxial nematic liquid crystals. The three chiral and 15 achiral constants are expressed as fourth-order polynomials in the order parameter $S$ and the degree of biaxiality $T$. Via the temperature dependence of the quantities $S$ and $T$ also the temperature dependence of the elastic constants is fixed.

Die elastischen Konstanten $K_{i j}$ der Frank-Oseen-Energiedichte für einachsige nematische Flüssigkristalle hängen über den Maier-Saupe-Ordnungsparameter von der Temperatur ab. Longa et al. haben kürzlich eine erweiterte Theorie nach Landau-Ginzburg-de Gennes benutzt, um die Funktionen $K_{i j}(S)$ bis zur vierten Ordnung nach $S$ zu entwickeln. Hier wird ein ähnliches Verfahren bei der elastischen Energiedichte von biaxialen nematischen Flüssigkristallen angewandt. Die drei chiralen und 15 nichtchiralen Konstanten werden als Polynome vierten Grades in Ordnungsparameter $S$ und Biaxialitätsparameter $T$ ausgedrückt. Durch deren Temperaturabhängigkeit ist damit das Temperaturverhalten der elastischen Konstanten bestimmt.

\section{Introduction}

For the description of most thermodynamic and elastic properties of nematic liquid crystals the quadrupole moment of the local angular distribution function of the molecules' long axes is sufficient. It is expressed by a symmetric traceless second rank tensor $\mathbf{Q}(\boldsymbol{r})=\left[Q_{i j}(\boldsymbol{r})\right], i, j=1,2,3$. The tensor serves as order parameter in a Landau free-energy density, which for example is used to investigate the transition from the isotropic phase to either the nematic, cholesteric or blue phases. The Landau freeenergy density usually is expanded into powers of $\mathbf{Q}$ and $\partial \mathbf{Q}=\left[\partial / \partial_{i} Q_{j k}\right]$ in a form going back to de Gennes [2]

$$
\begin{aligned}
f= & f_{\mathrm{b}}+f_{\mathrm{el}}, \\
f_{\mathrm{b}}= & A \operatorname{Tr} \mathbf{Q}^{2}+B \operatorname{Tr} \mathbf{Q}^{3}+C\left(\operatorname{Tr} \mathbf{Q}^{2}\right)^{2}, \\
f_{\mathrm{el}}= & L_{1}^{(2)} \partial_{i} Q_{j k} \partial_{i} Q_{j k}+L_{2}^{(2)} \partial_{i} Q_{j i} \partial_{k} Q_{j k}+ \\
& +L_{3}^{(2)} \partial_{i} Q_{j k} \partial_{j} Q_{i k}+L_{4}^{(2)} \varepsilon_{i j k} Q_{i l} \partial_{k} Q_{j l} .
\end{aligned}
$$

$f_{\mathrm{el}}$ are denoted "elastic terms" since their magnitude depends on the derivatives of the order parameter field, in contrast to $f_{\mathrm{b}}$, whose parts frequently are denoted "bulk terms".

1) Pfaffenwaldring 57, D-7000 Stuttgart 80, FRG. 
The quadrupolar tensor field which the system assumes at a certain temperature $t$ is found by minimizing the free energy

$$
F=\frac{1}{V} \int_{V} \mathrm{~d} \boldsymbol{r} f(\mathbf{Q}(\boldsymbol{r}), \partial \mathbf{Q}(\boldsymbol{r}))
$$

under appropriate boundary conditions. Temperature usually enters $F$ only in the most important bulk term $A \operatorname{Tr} \mathbf{Q}^{2}$ by a linear dependence $A=a\left(t-t^{*}\right)$, where $t^{*}$ is the temperature of absolute stability of the nematic phase.

One denotes as "hydrodynamic limit" the case, where the elastic contribution is much smaller than the bulk contribution. As rule of the thumb the limit is given, if the coherence length $\xi=\sqrt{L / A}$ is much smaller than the typical length scale $\lambda$ of the distortion. $L$ denotes the largest of the elastic constants $L_{i}^{(2)} . \lambda$ is, for instance, the wavelength where the Fourier amplitude of $\mathbf{Q}(\boldsymbol{r})$ is maximum. Thus $\xi$ is a measure of the weight of the elastic terms compared to the weight $A$ of the quadratic bulk form. Far away from the transition point $\xi$ is small and the hydrodynamic regime is the standard situation.

\section{Uniaxial Nematic Liquid Crystals}

In the hydrodynamic limit it is allowed first to minimize the bulk terms. In case of the expansion (2) the minimum of $f_{\mathrm{b}}$ always is a uniaxial tensor of the form

$$
Q(r)=S\left\{\hat{n}(r) \otimes \hat{n}(r)-\frac{1}{3}\right\}
$$

The bulk energy is degenerate with respect to the director $\hat{\boldsymbol{n}}$, i.e. on the set of unit vectors, where $\hat{\boldsymbol{n}}$ and $-\hat{\boldsymbol{n}}$ denote the same state (this set is the projective plane, $\boldsymbol{P}^{2}$ ). The energy is only dependent on the non-Goldstone variable $S$, the Maier-Saupe order parameter, which minimizes $f_{\mathrm{b}}$ with the value

$$
S(t)=-\frac{B}{4 C}\left(1+\sqrt{1-\frac{24 a\left(t-t^{*}\right) C}{B^{2}}}\right) .
$$

This equilibrium order parameter $S$ is assumed to be position independent in the hydrodynamic limit.

If the bulk solution (5) is inserted into the Landau-Ginzburg polynomial (1), the bulk energy is a constant, and the elastic energy turns into the Frank-Oseen (FO) energy density

$$
\begin{aligned}
f_{\mathrm{FO}}= & \frac{1}{2} K_{11}(\nabla \cdot \hat{\boldsymbol{n}})^{2}+\frac{1}{2} K_{22}\left(\hat{\boldsymbol{n}} \cdot \nabla \times \hat{\boldsymbol{n}}+q_{0}\right)^{2}+\frac{1}{2} K_{33}(\hat{\boldsymbol{n}} \times \nabla \times \hat{\boldsymbol{n}})^{2}+ \\
& +\frac{1}{2} K_{44} \nabla \cdot[(\hat{\boldsymbol{n}} \cdot \nabla) \hat{\boldsymbol{n}}-(\nabla \cdot \hat{\boldsymbol{n}}) \hat{\boldsymbol{n}}],
\end{aligned}
$$

expressed in terms of the "reduced" order parameter $\hat{\boldsymbol{n}}$ and its derivatives. The order parameter $S$ now enters the new effective elastic constants in the form

$$
\begin{aligned}
& K_{11}=2 S^{2}\left(2 L_{1}^{(2)}+L_{2}^{(2)}+L_{3}^{(2)}\right), \\
& K_{22}=2 S^{2}\left(2 L_{1}^{(2)}\right) \\
& K_{33}=2 S^{2}\left(2 L_{1}^{(2)}+L_{2}^{(2)}+L_{3}^{(2)}\right), \\
& K_{44}=2 S^{2}\left(2 L_{1}^{(2)}+L_{3}^{(2)}\right) .
\end{aligned}
$$


The chiral term is weighted by

$$
q_{0} K_{22}=S^{2} L_{4}^{(2)} \text {. }
$$

When $S$ is replaced by (6) we obtain a temperature dependence of the Frank-Oseen elastic constants $K_{i j}$.

Recently Longa et al. [1] used the angular momentum representation for the quadrupole tensor $\mathbf{Q}$ to extend $f_{\mathrm{el}}$ up to fourth order terms in $\mathbf{Q}$ and second order in its derivatives $\partial \mathbf{Q}$. The expansion is written in short

$$
f_{\mathrm{el}}=\sum_{n=2}^{4} \sum_{i=1}^{d_{n}} L_{i}^{(n)}\left[L_{i}^{(n)}\right]
$$

where $\left[L_{i}^{(n)}\right]$ stands for the $i$-th invariant of order $n$ in $\mathbf{Q}$ (Table 1 ) and $L_{i}^{(n)}$ denotes a corresponding temperature-independent elastic constant. If now the bulk solution (5) is inserted into $f_{\mathrm{el}}(10)$, the same form of the Frank-Oseen elastic energy is obtained as in (7), but with effective elastic constants which are higher order polynomials in $S$,

$$
K_{i j}=\sum_{n=2}^{4} S^{n} K_{i j}^{(n)} .
$$

Table 1

Independent elastic invariants constructed for nematics and cholesterics up to fourth order in the general biaxial order parameter $\mathbf{Q}$. Here we denote with an asterisk the $\mathrm{SO}(3)$-invariants, all other elastic terms are $\mathrm{O}(3)$-invariant

$$
\begin{aligned}
& \text { second order } \\
& {\left[L_{1}^{(2)}\right]=\partial_{i} Q_{j k} \partial_{i} Q_{j k}} \\
& {\left[L_{2}^{(2)}\right]=\partial_{i} Q_{j i} \partial_{k} Q_{j k}} \\
& {\left[L_{3}^{(2)}\right]=\partial_{i} Q_{j k} \partial_{j} Q_{i k}} \\
& { }^{*}\left[L_{4}^{(2)}\right]=\varepsilon_{i j k} Q_{i l} \partial_{j} Q_{k l}
\end{aligned}
$$

third order

$$
\begin{aligned}
{\left[L_{1}^{(3)}\right] } & =Q_{i j} \partial_{k} Q_{i j} \partial_{l} Q_{k l} \\
{\left[L_{3}^{(3)}\right] } & =Q_{i j} \partial_{k} Q_{i k} \partial_{l} Q_{j l} \\
{\left[L_{5}^{(3)}\right] } & =Q_{i j} \partial_{l} Q_{i k} \partial_{k} Q_{j l} \\
*\left[L_{7}^{(3)}\right] & =\varepsilon_{i j k} Q_{i l} Q_{j m} \partial_{m} Q_{k l}
\end{aligned}
$$$$
\left[L_{2}^{(3)}\right]=Q_{i j} \partial_{j} Q_{i k} \partial_{l} Q_{k l}
$$$$
\left[L_{4}^{(3)}\right]=Q_{i j} \partial_{l} Q_{i k} \partial_{l} Q_{j k}
$$$$
\left[L_{6}^{(3)}\right]=Q_{i j} \partial_{l} Q_{i k} \partial_{j} Q_{k l}
$$

fourth order

$$
\begin{aligned}
& {\left[L_{1}^{(4)}\right]=Q_{i j} Q_{i j} \partial_{k} Q_{k l} \partial_{m} Q_{l m}} \\
& {\left[L_{3}^{(4)}\right]=Q_{i j} Q_{i j} \partial_{k} Q_{l m} \partial_{m} Q_{k l}} \\
& {\left[L_{5}^{(4)}\right]=Q_{i k} Q_{j k} \partial_{j} Q_{i l} \partial_{m} Q_{l m}} \\
& {\left[L_{7}^{(4)}\right]=Q_{i k} Q_{j k} \partial_{m} Q_{i l} \partial_{m} Q_{j l}} \\
& {\left[L_{9}^{(4)}\right]=Q_{i k} Q_{j k} \partial_{m} Q_{i l} \partial_{j} Q_{l m}} \\
& {\left[L_{11}^{(4)}\right]=Q_{i j} Q_{k l} \partial_{i} Q_{l m} \partial_{j} Q_{k m}} \\
& {\left[L_{13}^{(4)}\right]=Q_{i j} Q_{k l} \partial_{m} Q_{i j} \partial_{m} Q_{k l}} \\
& *\left[L_{15}^{(4)}\right]=\varepsilon_{i j k} Q_{l m} Q_{l m} Q_{i n} \partial_{j} Q_{k n}
\end{aligned}
$$$$
\left[L_{2}^{(4)}\right]=Q_{i j} Q_{i j} \partial_{k} Q_{l m} \partial_{k} Q_{l m}
$$$$
\left[L_{4}^{(4)}\right]=Q_{i k} Q_{j k} \partial_{l} Q_{i j} \partial_{m} Q_{l m}
$$$$
\left[L_{6}^{(4)}\right]=Q_{i k} Q_{j k} \partial_{l} Q_{i l} \partial_{m} Q_{j m}
$$$$
\left[L_{8}^{(4)}\right]=Q_{i k} Q_{j k} \partial_{m} Q_{i l} \partial_{l} Q_{j m}
$$$$
\left[L_{10}^{(4)}\right]=Q_{i j} Q_{k l} \partial_{i} Q_{j l} \partial_{m} Q_{k m}
$$$$
\left[L_{12}^{(\mathbf{4})}\right]=Q_{i j} Q_{k l} \partial_{m} Q_{i j} \partial_{l} Q_{k m}
$$$$
*\left[L_{14}^{(4)}\right]=\varepsilon_{i j k} Q_{i l} Q_{j m} Q_{m n} \partial_{k} Q_{l n}
$$ 
The coefficients $K_{i j}^{(2)}$ are identical to the coefficients $K_{i j} / S^{2}$ of $(8)$ and $K_{i j}^{(n)}, n \geqq 3$, are the polynomial coefficients

$$
\begin{aligned}
& K_{11}^{(3)}=\frac{2}{3}\left(-L_{2}^{(3)}+2 L_{3}^{(3)}+L_{4}^{(3)}+2 L_{5}^{(3)}-L_{6}^{(3)}\right), \\
& K_{22}^{(3)}=\frac{2}{3} L_{4}^{(3)}, \\
& K_{33}^{(3)}=\frac{2}{3}\left(2 L_{2}^{(3)}-L_{3}^{(3)}+L_{4}^{(3)}-L_{5}^{(3)}+2 L_{6}^{(3)}\right), \\
& K_{44}^{(3)}=\frac{2}{3}\left(L_{4}^{(3)}+2 L_{5}^{(3)}-L_{6}^{(3)}\right)
\end{aligned}
$$

for third order, and

$$
\begin{aligned}
K_{11}^{(4)}= & \frac{2}{9}\left(6 L_{1}^{(4)}+12 L_{2}^{(4)}+6 L_{3}^{(4)}+L_{5}^{(4)}+4 L_{6}^{(4)}+5 L_{7}^{(4)}+\right. \\
& \left.+4 L_{8}^{(4)}+L_{9}^{(4)}-2 L_{10}^{(4)}-L_{11}^{(4)}\right) \\
K_{22}^{(4)}= & \frac{2}{9}\left(12 L_{2}^{(4)}+5 L_{7}^{(4)}-L_{11}^{(4)}\right) \\
K_{33}^{(4)}= & \frac{2}{9}\left(6 L_{1}^{(4)}+12 L_{2}^{(4)}+6 L_{3}^{(4)}+4 L_{5}^{(4)}+L_{6}^{(4)}+5 L_{7}^{(4)}+\right. \\
& +L_{8}^{(4)}+4 L_{9}^{(4)}-2 L_{10}^{(4)}+2 L_{11}^{(4)} \\
K_{44}^{(4)}= & \frac{2}{9}\left(12 L_{2}^{(4)}+6 L_{3}^{(4)}+5 L_{7}^{(4)}+4 L_{8}^{(4)}+L_{9}^{(4)}-L_{11}^{(4)}\right)
\end{aligned}
$$

for fourth order contributions. The chiral elastic constant reads

$$
q_{0} K_{22}=S^{2} L_{4}^{(2)}-S^{3} \frac{L_{7}^{(3)}}{3}-S^{4} \frac{2}{3}\left(\frac{L_{14}^{(14)}}{3}-L_{15}^{(4)}\right) .
$$

\begin{tabular}{|c|c|c|c|c|c|c|c|c|c|}
\hline \multirow[t]{2}{*}{$K_{j k}$} & \multicolumn{3}{|c|}{$p_{1, j k}^{(2)}(S, T)$} & \multicolumn{3}{|c|}{$p_{2, j k}^{(2)}(S, T)$} & \multicolumn{3}{|c|}{$p_{3, j k}^{(2)}(S, T)$} \\
\hline & $S^{2}$ & $S T$ & $T^{2}$ & $S^{2}$ & $S T$ & $T^{2}$ & $S^{2}$ & $S T$ & $T^{2}$ \\
\hline$K_{l 1}$ & 0 & -96 & 32 & 0 & 0 & 64 & 0 & -24 & 24 \\
\hline$K_{l 2}$ & 0 & -96 & 32 & 0 & 0 & 0 & 0 & 0 & 0 \\
\hline$K_{l 3}$ & 0 & -96 & 32 & 0 & 48 & 16 & 0 & 0 & 16 \\
\hline$K_{l 4}$ & 0 & -96 & 32 & 0 & 0 & 0 & 0 & -48 & 16 \\
\hline$K_{l m}$ & 0 & 0 & 0 & 0 & -48 & 16 & 0 & -24 & 8 \\
\hline$K_{m 1}$ & 0 & 96 & 32 & 0 & 96 & 0 & 0 & 72 & 8 \\
\hline$K_{m 2}$ & 0 & 96 & 32 & 0 & 0 & 0 & 0 & 0 & 0 \\
\hline$K_{m 3}$ & 0 & 96 & 32 & 0 & 0 & 0 & 0 & 24 & 8 \\
\hline$K_{m 4}$ & 0 & 96 & 32 & 0 & 0 & 0 & 0 & 48 & 16 \\
\hline$K_{m n}$ & 0 & 0 & 0 & 0 & 96 & 0 & 0 & 48 & 0 \\
\hline$K_{n 1}$ & 144 & 0 & -16 & 72 & -96 & -8 & 72 & -48 & -8 \\
\hline$K_{n 2}$ & 144 & 0 & -16 & 0 & 0 & 0 & 0 & 0 & 0 \\
\hline$K_{n 3}$ & 144 & 0 & -16 & 72 & -48 & 8 & 72 & -24 & 0 \\
\hline$K_{n 3}$ & 144 & 0 & -16 & 0 & 0 & 0 & 72 & 0 & -8 \\
\hline$K_{n l}$ & 0 & 0 & 0 & 0 & -48 & -16 & 0 & -24 & -8 \\
\hline
\end{tabular}

The higher order expansion also lifts the degeneracy of the elastic constants $K_{11}$ and $K_{33}(8)$. The constants $L_{i}^{(n)}$ can be derived from the temperature dependence of the $K_{i j}$.

Of course the Frank-Oseen energy can be constructed also by pure symmetry arguments. In the sense of invariant theory the $K_{i j}$ simply are weights of the invariant expressions for splay, bend, twist and surface terms, and do not give access to the temperature dependence of the elastic constants.

Table 2

Coefficients of the monomials $S^{n} T^{m}$ with $n+m=2$ in the polynomials $p_{i, j k}^{(2)}(S, T)$, $i=1,2,3$, of $(18)$ 


\section{Biaxial Nematic Liquid Crystals}

If $f_{\mathrm{b}}$ is extended to sixth order [6]

$$
\begin{aligned}
f_{\mathrm{b}}= & A \operatorname{Tr} \mathbf{Q}^{2}+B \operatorname{Tr} \mathbf{Q}^{3}+C\left(\operatorname{Tr} \mathbf{Q}^{2}\right)^{2}+D \operatorname{Tr} \mathbf{Q}^{2} \operatorname{Tr} \mathbf{Q}^{3}+ \\
& +E^{\prime}\left(\operatorname{Tr} \mathbf{Q}^{2}\right)^{3}+E^{\prime \prime}\left(\operatorname{Tr} \mathbf{Q}^{3}\right)^{2},
\end{aligned}
$$

Table 3

Coefficients of the monomials $S^{n} T^{m}$ with $n+m=3$ in the polynomials $p_{i, j k}^{(3)}(S, T)$, $i=1, \ldots, 6$. The LGdG invariant $\left[L_{1}^{(3)}\right]$ vanishes in the hard biaxial phase, so that

\begin{tabular}{|c|c|c|c|c|c|c|c|c|}
\hline \multirow[t]{2}{*}{$K_{j k}$} & \multicolumn{2}{|c|}{$p_{2, j k}^{(3)}(S, T)$} & \multirow[b]{2}{*}{$S T^{2}$} & \multirow[b]{2}{*}{$T^{3}$} & \multicolumn{2}{|c|}{$p_{3, j k}^{(3)}(S, T)$} & \multirow[b]{2}{*}{$S T^{2}$} & \multirow[b]{2}{*}{$T^{3}$} \\
\hline & $S^{3}$ & $S^{2} T$ & & & $S^{3}$ & $S^{2} T$ & & \\
\hline$K_{l 1}$ & 0 & -144 & -16 & -32 & 0 & 144 & -64 & 48 \\
\hline$K_{l 2}$ & 0 & 0 & 0 & 0 & 0 & 0 & 0 & 0 \\
\hline$K_{l 3}$ & 0 & 48 & -128 & -16 & 0 & 96 & -128 & 32 \\
\hline$K_{l 4}$ & 0 & 0 & 0 & 0 & 0 & 0 & 0 & 0 \\
\hline$K_{l m}$ & 0 & -192 & -80 & 48 & 0 & 240 & 64 & -48 \\
\hline$K_{m 1}$ & 0 & -48 & 144 & 0 & 0 & -48 & -192 & -16 \\
\hline$K_{m 2}$ & 0 & 0 & 0 & 0 & 0 & 0 & 0 & 0 \\
\hline$K_{m 3}$ & 0 & 144 & -48 & -32 & 0 & -144 & 0 & 16 \\
\hline$K_{m 4}$ & 0 & 0 & 0 & 0 & 0 & 0 & 0 & 0 \\
\hline$K_{m n}$ & 0 & -480 & 0 & 0 & 0 & 384 & 0 & 0 \\
\hline$K_{n 1}$ & -144 & 192 & 16 & 32 & 288 & -96 & -32 & -32 \\
\hline$K_{n 2}$ & 0 & 0 & 0 & 0 & 0 & 0 & 0 & 0 \\
\hline$K_{n 3}$ & 288 & 240 & -112 & 0 & -144 & -192 & 80 & 0 \\
\hline$K_{n 4}$ & 0 & 0 & 0 & 0 & 0 & 0 & 0 & 0 \\
\hline$K_{n l}$ & 0 & -192 & 80 & 48 & 0 & 240 & -64 & -48 \\
\hline
\end{tabular}
$p_{1, j, j k}^{(3)}(S, T)=0$, for all $j$ and $k$

\begin{tabular}{|c|c|c|c|c|c|c|c|c|}
\hline \multirow[t]{2}{*}{$K_{j k}$} & \multicolumn{4}{|c|}{$p_{4, j k}^{(3)}(S, T)$} & \multicolumn{4}{|c|}{$p_{5, j k}^{(3)}(S, T)$} \\
\hline & $S^{3}$ & $S^{2} T$ & $S T^{2}$ & $T^{3}$ & $S^{3}$ & $S^{2} T$ & $S T^{2}$ & $T^{3}$ \\
\hline$K_{l 1}$ & 0 & 48 & -64 & 16 & 0 & -24 & -156 & 36 \\
\hline$K_{l 2}$ & 0 & 48 & -64 & 16 & 0 & 0 & 0 & 0 \\
\hline$K_{l 3}$ & 0 & 48 & -64 & 16 & 0 & -432 & 64 & 8 \\
\hline$K_{l 4}$ & 0 & 48 & -64 & 16 & 0 & 96 & -128 & 32 \\
\hline$K_{l m}$ & 0 & 0 & 0 & 0 & 0 & 408 & -28 & -36 \\
\hline$K_{m 1}$ & 0 & -48 & -64 & -16 & 0 & -216 & -100 & -28 \\
\hline$K_{m 2}$ & 0 & -48 & -64 & -16 & 0 & 0 & 0 & 0 \\
\hline $\boldsymbol{K}_{m 3}$ & 0 & -48 & -64 & -16 & 0 & 24 & 92 & 28 \\
\hline$K_{m 4}$ & 0 & -48 & -64 & -16 & 0 & -96 & -128 & -32 \\
\hline$K_{m n}$ & 0 & 0 & 0 & 0 & 0 & 48 & 0 & -24 \\
\hline$K_{n \mathrm{1}}$ & 144 & 0 & -16 & 0 & 288 & 240 & -32 & -8 \\
\hline$K_{n 2}$ & 144 & 0 & -16 & 0 & 0 & 0 & 0 & 0 \\
\hline$K_{n 3}$ & 144 & 0 & -16 & 0 & -144 & -24 & -12 & 12 \\
\hline$K_{n 4}$ & 144 & 0 & -16 & 0 & 288 & 0 & -32 & 0 \\
\hline$K_{n l}$ & 0 & 0 & 0 & 0 & 0 & 408 & 28 & -36 \\
\hline
\end{tabular}


Table 3 (continued)

\begin{tabular}{lrrrr}
\hline \multirow{2}{*}{$K_{j k}$} & \multicolumn{2}{c}{$p_{6, j k}^{(3)}(S, T)$} & & \\
\cline { 2 - 5 } & \multicolumn{1}{c}{$S^{3}$} & $S^{2} T$ & $S T^{2}$ & $T^{3}$ \\
\hline$K_{l 1}$ & 0 & 48 & 84 & -20 \\
$K_{l 2}$ & 0 & 0 & 0 & 0 \\
$K_{l 3}$ & 0 & 432 & -128 & 8 \\
$K_{l 4}$ & 0 & -48 & 64 & -16 \\
$K_{l m}$ & 0 & -384 & 20 & 36 \\
$K_{m 1}$ & 0 & 144 & 44 & 12 \\
$K_{m 2}$ & 0 & 0 & 0 & 0 \\
$K_{m 3}$ & 0 & -48 & -148 & -44 \\
$K_{m 4}$ & 0 & 48 & 64 & 16 \\
$K_{m n 2}$ & 0 & -96 & 0 & 24 \\
$K_{n 1}$ & -144 & -192 & 16 & 8 \\
$K_{n 2}$ & 0 & 0 & 0 & 0 \\
$K_{n 3}$ & 288 & 48 & -12 & -12 \\
$K_{n 4}$ & -144 & 0 & 16 & 0 \\
$K_{n l}$ & 0 & -384 & -20 & 36 \\
\hline
\end{tabular}

then in parameter space $\left\{A, B, C, D, E^{\prime}, E^{\prime \prime}\right\}$ there are regions where $f_{\mathrm{b}}$ is minimized by a biaxial order parameter of the form

$$
\mathbf{Q}(\boldsymbol{r})=S\left\{\hat{\boldsymbol{n}}(\boldsymbol{r}) \otimes \hat{\boldsymbol{n}}(\boldsymbol{r})-\frac{1}{3}\right\}+\frac{T}{3}\{\hat{\boldsymbol{l}}(\boldsymbol{r}) \otimes \hat{\boldsymbol{l}}(\boldsymbol{r})-\hat{\boldsymbol{m}}(\boldsymbol{r}) \otimes \hat{\boldsymbol{m}}(\boldsymbol{r})\} .
$$

$f_{\mathrm{b}}$ is degenerate with respect to the orientations of the orthonormal tripod $\{\hat{\boldsymbol{u}}(\boldsymbol{r})$ $\hat{\boldsymbol{m}}(\boldsymbol{r}), \hat{\boldsymbol{n}}(\boldsymbol{r})\}$, forming the orthogonal group $\mathrm{SO}(3)$. The phase, in which the Goldstone variables $\{\hat{\boldsymbol{l}}(\boldsymbol{r}), \hat{\boldsymbol{m}}(\boldsymbol{r}), \hat{\boldsymbol{n}}(\boldsymbol{r})\}$ vary in space, but the order parameter $S$ and the degree of biaxiality $T$ are constant, is denoted "hard biaxial nematic phase" [7]. If ansatz (16) is inserted into the extended Landau-Ginzburg-de Gennes (LGdG) elastic energy density $f_{\mathrm{el}}(10)$, then a generalized Frank-Oseen elastic energy for hard biaxial nematic liquid crystals results.

This energy was derived by Trebin [8], Brand and Pleiner [9], and Govers and Vertogen $[10,11]$ by symmetry considerations and has the form (according to [11])

$$
\begin{aligned}
f_{\mathrm{el}}^{\mathrm{biax}}= & k_{l}(\hat{\boldsymbol{l}} \cdot \nabla \times \hat{\boldsymbol{l}})+k_{m}(\hat{\boldsymbol{m}} \cdot \nabla \times \hat{\boldsymbol{m}})+k_{n}(\hat{\boldsymbol{n}} \cdot \nabla \times \hat{\boldsymbol{n}})+ \\
& +\frac{1}{2} K_{l 1}(\nabla \cdot \hat{\boldsymbol{l}})^{2}+\frac{1}{2} K_{l 2}(\hat{\boldsymbol{l}} \cdot \nabla \times \hat{\boldsymbol{l}})^{2}+\frac{1}{2} K_{l 3}(\hat{\boldsymbol{l}} \times \Delta \times \hat{\boldsymbol{l}})^{2}+ \\
& +\frac{1}{2} K_{l 4} \nabla \cdot[(\hat{\boldsymbol{l}} \cdot \nabla) \hat{\boldsymbol{l}}-(\nabla \cdot \hat{\boldsymbol{l}} \hat{\boldsymbol{l}}]+ \\
& +\frac{1}{2} K_{m 1}(\nabla \cdot \hat{\boldsymbol{m}})^{2}+\frac{1}{2} K_{m 2}(\hat{\boldsymbol{m}} \cdot \nabla \times \hat{\boldsymbol{m}})^{2}+\frac{1}{2} K_{m 3}(\hat{\boldsymbol{m}} \times \nabla \times \hat{\boldsymbol{m}})^{2}+ \\
& +\frac{1}{2} K_{m 4} \nabla \cdot[(\hat{\boldsymbol{m}} \cdot \nabla) \hat{\boldsymbol{m}}-(\nabla \cdot \hat{\boldsymbol{m}}) \hat{\boldsymbol{m}}]+ \\
& +\frac{1}{2} K_{n 1}(\nabla \cdot \hat{\boldsymbol{n}})^{2}+\frac{1}{2} K_{n 2}(\hat{\boldsymbol{n}} \cdot \nabla \times \hat{\boldsymbol{n}})^{2}+\frac{1}{2} K_{n 3}(\hat{\boldsymbol{n}} \times \nabla \times \hat{\boldsymbol{n}})^{2}+ \\
& +\frac{1}{2} K_{n 4} \nabla \cdot[(\hat{\boldsymbol{n}} \cdot \nabla) \hat{\boldsymbol{n}}-(\nabla \cdot \hat{\boldsymbol{n}}) \hat{\boldsymbol{n}}]+ \\
& +\frac{1}{2} K_{l m}(\hat{\boldsymbol{l}} \cdot \nabla \times \hat{\boldsymbol{m}})^{2}+\frac{1}{2} K_{m n}(\hat{\boldsymbol{m}} \cdot \nabla \times \hat{\boldsymbol{n}})^{2}+\frac{1}{2} K_{n l}(\hat{\boldsymbol{n}} \cdot \nabla \times \hat{\boldsymbol{l}})^{2}
\end{aligned}
$$

with 15 nematic and 3 chiral-nematic contributions. 
Use of the generalized LGdG energy now allows to express the biaxial elastic constants in terms of the order parameters $S$ and $T$

$$
K_{j k}(S, T)=\sum_{n=2}^{4}\left(\frac{1}{6}\right)^{n}\left\{\sum_{i=1}^{d_{n}} p_{i, j k}^{(n)}(S, T) L_{i}^{(n)}\right\},
$$

where $p_{i, j k}^{(n)}(S, T)$ are homogeneous polynomials of degree $n$ in $S$ and $T$. The coefficients of the monomials $S^{m} T^{p}$ with $m+p=n$ in $p_{i, j k}^{(n)}(S, T)$, multiplying the LGdG elastic constants $L_{i}^{(n)}$, are presented in Tables 2, 3, and 4. The three chiral-nematic constants $k_{l}, k_{m}$, and $k_{n}$ are listed as functions of $S$ and $T$ in Table 5 .

Table 4

Coefficients of the monomials $S^{n} T^{m}$ with $n+m=4$ in the polynomials $p_{i, j k}^{(4)}(S, T)$, $i=1, \ldots, 13$. The $L G d G$ invariants $\left[L_{4}^{(4)}\right]$, and $\left[L_{12}^{(4)}\right]$, and $\left[L_{13}^{(4)}\right]$ vanish in the hard biaxial phase, so that $p_{4, j k}^{(4)}(S, T)=0, p_{12, j k}^{(4)}(S, T)=0$, and $p_{13, j k}(S, T)=0$ for all $j$ and $k$

\begin{tabular}{|c|c|c|c|c|c|c|c|c|c|c|}
\hline \multirow[t]{2}{*}{$K_{j k}$} & \multicolumn{5}{|c|}{$p_{1, j k}^{(4)}(S, T)$} & \multicolumn{5}{|c|}{$p_{2, j k}^{(4)}(S, T)$} \\
\hline & $S^{4}$ & $S^{3} T$ & $S^{2} T^{2}$ & $S T^{3}$ & $T^{4}$ & $S^{4}$ & $S^{3} T$ & $S^{2} T^{2}$ & $S T^{3}$ & $T^{4}$ \\
\hline$K_{l 1}$ & 0 & 0 & 768 & 0 & 0 & 0 & -2304 & 768 & -768 & 256 \\
\hline$K_{l 2}$ & 0 & 0 & 0 & 0 & 0 & 0 & -2304 & 768 & -768 & 256 \\
\hline$K_{l 3}$ & 0 & 1152 & 384 & 384 & 128 & 0 & -2304 & 768 & -768 & 256 \\
\hline$K_{l 4}$ & 0 & 0 & 0 & 0 & 0 & 0 & -2304 & 768 & -768 & 256 \\
\hline$K_{l m}$ & 0 & -1152 & 384 & -384 & 128 & 0 & 0 & 0 & 0 & 0 \\
\hline$K_{m 1}$ & 0 & 2304 & 0 & 768 & 0 & 0 & 2304 & 768 & 768 & 256 \\
\hline$K_{m 2}$ & 0 & 0 & 0 & 0 & 0 & 0 & 2304 & 768 & 768 & 256 \\
\hline$K_{m 3}$ & 0 & 0 & 0 & 0 & 0 & 0 & 2304 & 768 & 768 & 256 \\
\hline$K_{m 4}$ & 0 & 0 & 0 & 0 & 0 & 0 & 2304 & 768 & 768 & 256 \\
\hline$K_{m n}$ & 0 & 2304 & 0 & 768 & 0 & 0 & 0 & 0 & 0 & 0 \\
\hline$K_{n 1}$ & 1728 & -2304 & 384 & -768 & -64 & 3456 & 0 & 768 & 0 & -128 \\
\hline$K_{n 2}$ & 0 & 0 & 0 & 0 & 0 & 3456 & 0 & 768 & 0 & -128 \\
\hline$K_{n 3}$ & 1728 & -1152 & 768 & -384 & 64 & 3456 & 0 & 768 & 0 & -128 \\
\hline$K_{n 4}$ & 0 & 0 & 0 & 0 & 0 & 3456 & 0 & 768 & 0 & -128 \\
\hline$\underline{K_{n l}}$ & 0 & -1152 & -384 & -384 & -128 & 0 & 0 & 0 & 0 & 0 \\
\hline \multirow[t]{2}{*}{$K_{j k}$} & \multicolumn{5}{|c|}{$p_{3, j k}^{(4)}(S, T)$} & \multicolumn{5}{|c|}{$p_{5, j k}^{(4)}(S, T)$} \\
\hline & $S^{4}$ & $S^{3} T$ & $S^{2} T^{2}$ & $S T^{3}$ & $T^{4}$ & $S^{4}$ & $S^{3} T$ & $S^{2} T^{2}$ & $S T^{3}$ & $T^{4}$ \\
\hline$K_{l 1}$ & 0 & -576 & 576 & -192 & 192 & 0 & -288 & -24 & 224 & 128 \\
\hline$K_{l 2}$ & 0 & 0 & 0 & 0 & 0 & 0 & 0 & 0 & 0 & 0 \\
\hline$K_{l 3}$ & 0 & 0 & 384 & 0 & 128 & 0 & 480 & 160 & 160 & 96 \\
\hline$K_{l 4}$ & 0 & -1152 & 384 & -384 & 128 & 0 & 0 & 0 & 0 & 0 \\
\hline$K_{l m}$ & 0 & -576 & 192 & -192 & 64 & 0 & -768 & 544 & -192 & 32 \\
\hline $\boldsymbol{K}_{m \mathbf{1}}$ & 0 & 1728 & 192 & 576 & 64 & 0 & 1536 & -288 & 192 & -32 \\
\hline$K_{m 2}$ & 0 & 0 & 0 & 0 & 0 & 0 & 0 & 0 & 0 & 0 \\
\hline$K_{m 3}$ & 0 & 576 & 192 & 192 & 64 & 0 & 288 & 192 & 32 & 0 \\
\hline$K_{m 4}$ & 0 & 1152 & 384 & 384 & 128 & 0 & 0 & 0 & 0 & 0 \\
\hline$K_{m n}$ & 0 & 1152 & 0 & 384 & 0 & 0 & 672 & -288 & 480 & 32 \\
\hline$K_{n 1}$ & 1728 & -1152 & 384 & -384 & -64 & 288 & -1248 & 544 & -416 & -64 \\
\hline$K_{n 2}$ & 0 & 0 & 0 & 0 & 0 & 0 & 0 & 0 & 0 & 0 \\
\hline$K_{n 3}$ & 1728 & -576 & 576 & -192 & 0 & 1152 & -768 & 416 & -192 & 32 \\
\hline$K_{n 4}$ & -1728 & 1152 & -768 & 384 & -64 & 0 & 0 & 0 & 0 & 0 \\
\hline$K_{n l}$ & 0 & -576 & -192 & -192 & -64 & 0 & 96 & -256 & -288 & -64 \\
\hline
\end{tabular}




\begin{tabular}{|c|c|c|c|c|c|c|c|c|c|c|}
\hline \multirow[t]{2}{*}{$K_{j k}$} & \multicolumn{5}{|c|}{$p_{6, j k}^{(4)}(S, T)$} & \multicolumn{5}{|c|}{$p_{7, j k}^{(4)}(S, T)$} \\
\hline & $S^{4}$ & $S^{3} T$ & $S^{2} T^{2}$ & $S T^{3}$ & $T^{4}$ & $S^{4}$ & $S^{3} T$ & $S^{2} T^{2}$ & $S T^{3}$ & $T^{4}$ \\
\hline$K_{l 1}$ & 0 & -576 & -160 & -192 & 160 & 0 & -1536 & 128 & -256 & 128 \\
\hline$K_{l 2}$ & 0 & 0 & 0 & 0 & 0 & 0 & -1536 & 128 & -256 & 128 \\
\hline$K_{l 3}$ & 0 & 192 & -320 & 64 & 64 & 0 & -1536 & 128 & -256 & 128 \\
\hline$K_{l 4}$ & 0 & 0 & 0 & 0 & 0 & 0 & -1536 & 128 & -256 & 128 \\
\hline$K_{l m}$ & 0 & -768 & 544 & 0 & -32 & 0 & 0 & 0 & 0 & 0 \\
\hline$K_{m 1}$ & 0 & 1536 & 480 & 256 & 32 & 0 & 1536 & 0 & 256 & 128 \\
\hline$K_{m 2}$ & 0 & 0 & 0 & 0 & 0 & 0 & 1536 & 0 & 256 & 128 \\
\hline$K_{m 3}$ & 0 & 576 & 288 & -64 & -32 & 0 & 1536 & 0 & 256 & 128 \\
\hline$K_{m 4}$ & 0 & 0 & 0 & 0 & 0 & 0 & 1536 & 0 & 256 & 128 \\
\hline$K_{m n}$ & 0 & -192 & -576 & 192 & 64 & 0 & 0 & 0 & 0 & 0 \\
\hline$K_{n 1}$ & 1152 & -960 & 448 & -64 & -64 & 1440 & 0 & 704 & 0 & -96 \\
\hline$K_{n 2}$ & 0 & 0 & 0 & 0 & 0 & 1440 & 0 & 704 & 0 & -96 \\
\hline$K_{n 3}$ & 288 & -768 & 224 & 0 & 0 & 1440 & 0 & 704 & 0 & -96 \\
\hline$K_{n 4}$ & 0 & 0 & 0 & 0 & 0 & 1440 & 0 & 704 & 0 & -96 \\
\hline$K_{n l}$ & 0 & 960 & 32 & -192 & -32 & 0 & 0 & 0 & 0 & 0 \\
\hline \multirow[t]{2}{*}{$K_{j k}$} & \multicolumn{5}{|c|}{$p_{8, j k}^{(4)}(S, T)$} & \multicolumn{5}{|c|}{$p_{9, j k}^{(4)}(S, T)$} \\
\hline & $S^{4}$ & $S^{3} T$ & $S^{2} T^{2}$ & $S T^{3}$ & $T^{4}$ & $S^{4}$ & $S^{3} T$ & $S^{2} T^{2}$ & $S T^{3}$ & $T^{4}$ \\
\hline$K_{l 1}$ & 0 & -1104 & 456 & -144 & 88 & 0 & -744 & 0 & 200 & 96 \\
\hline$K_{l 2}$ & 0 & 0 & 0 & 0 & 0 & 0 & 0 & 0 & 0 & 0 \\
\hline$K_{l 3}$ & 0 & -864 & -320 & 160 & 64 & 0 & -432 & 160 & 112 & 96 \\
\hline$K_{l 4}$ & 0 & -992 & 448 & -320 & 64 & 0 & -480 & -32 & -32 & 32 \\
\hline$K_{l m}$ & 0 & -240 & 1160 & -48 & -104 & 0 & -312 & 608 & -168 & 0 \\
\hline$K_{m 1}$ & 0 & 1008 & -136 & 304 & 104 & 0 & 1080 & -352 & $\mathrm{I} 68$ & 0 \\
\hline$K_{m 2}$ & 0 & 0 & 0 & 0 & 0 & 0 & 0 & 0 & 0 & 0 \\
\hline$K_{m 3}$ & 0 & 1104 & -328 & -112 & 40 & 0 & 744 & 128 & 56 & 32 \\
\hline$K_{m 4}$ & 0 & 192 & 448 & 320 & 64 & 0 & 480 & -32 & 32 & 32 \\
\hline$K_{m n}$ & 0 & -1248 & -576 & 288 & 64 & 0 & -240 & -288 & 432 & 32 \\
\hline$K_{n 1}$ & 1152 & 96 & 448 & -160 & -64 & 288 & -336 & 544 & -368 & -64 \\
\hline$K_{n 2}$ & 0 & 0 & 0 & 0 & 0 & 0 & 0 & 0 & 0 & 0 \\
\hline$K_{n 3}$ & 288 & -240 & 840 & -48 & -72 & 1152 & -312 & 480 & -168 & \\
\hline$K_{n 4}$ & 1152 & 0 & -128 & 0 & 0 & 288 & 0 & 256 & 0 & -32 \\
\hline$K_{n l}$ & 0 & 1488 & -584 & -240 & 40 & 0 & 552 & -320 & -264 & -32 \\
\hline \multirow[t]{2}{*}{$K_{j k}$} & \multicolumn{5}{|c|}{$p_{10, j k}^{(4)}(S, T)$} & \multicolumn{5}{|c|}{$p_{11, j k}^{(4)}(S, T)$} \\
\hline & $S^{4}$ & $S^{3} T$ & $S^{2} T^{2}$ & $S T^{\mathbf{3}}$ & $T^{4}$ & $S^{4}$ & $S^{3} T$ & $S^{2} T^{2}$ & $S T^{3}$ & $T^{4}$ \\
\hline$K_{l 1}$ & & 864 & 320 & -128 & -160 & 0 & 624 & -232 & -48 & -24 \\
\hline$K_{l 2}$ & 0 & 0 & 0 & 0 & 0 & 0 & -384 & -256 & 128 & 0 \\
\hline$K_{l 3}$ & 0 & $-\quad 96$ & 352 & -224 & -96 & 0 & 480 & 512 & -352 & 0 \\
\hline$K_{l 4}$ & 0 & 0 & 0 & 0 & 0 & 0 & -384 & -256 & 128 & 0 \\
\hline$K_{l m}$ & 0 & 960 & -1184 & : 96 & 64 & 0 & 144 & -1128 & 48 & 104 \\
\hline$K_{m \mathrm{I}}$ & 0 & -1920 & 96 & -160 & 0 & 0 & -336 & 296 & -112 & -40 \\
\hline$K_{m 2}$ & 0 & 0 & 0 & 0 & 0 & 0 & 384 & -256 & -128 & 0 \\
\hline$K_{m 3}$ & 0 & -864 & -192 & 128 & 32 & 0 & -624 & 488 & 304 & 24 \\
\hline$K_{m \mathbf{4}}$ & 0 & 0 & 0 & 0 & 0 & 0 & 384 & -256 & -128 & 0 \\
\hline$K_{m n}$ & 0 & 672 & 864 & -480 & -96 & 0 & 1440 & $\mathbf{5 7 6}$ & -288 & -64 \\
\hline$K_{n \mathbf{1}}$ & -576 & 1056 & -800 & 288 & 96 & -288 & -288 & -256 & 160 & 32 \\
\hline$K_{n 2}$ & 0 & 0 & 0 & 0 & 0 & -288 & 0 & 320 & 0 & -32 \\
\hline$K_{n 3}$ & -576 & 960 & -544 & 96 & 0 & 576 & 144 & -616 & 48 & 40 \\
\hline$K_{n 4}$ & 0 & 0 & 0 & 0 & 0 & -288 & 0 & 320 & 0 & -32 \\
\hline$K_{n l}$ & 0 & -1632 & 320 & 384 & 32 & 0 & -1584 & 552 & 240 & -40 \\
\hline
\end{tabular}


Table 5

Coefficients of the three chiral strain modes $\hat{\boldsymbol{i}} \cdot \nabla \times \hat{\boldsymbol{i}}, \hat{\boldsymbol{m}} \cdot \nabla \times \hat{\boldsymbol{m}}$ and $\hat{\boldsymbol{n}} \cdot \nabla \times \hat{\boldsymbol{n}}$ in terms of the $\mathrm{LGdG}$ elastic constants

$$
\begin{gathered}
k_{l}=-2 T(T-3 S)\left\{-\frac{L_{4}^{(2)}}{9}-\frac{L_{7}^{(3)}}{27} \frac{(S-T)}{2}+\frac{L_{14}^{(4)}}{81}\left(T^{2}+2 S T+5 S^{2}\right)-\right. \\
\left.-\frac{L_{15}^{(4)}}{81}\left(2 T^{2}+6 S^{2}\right)\right\} \\
k_{m}=-2 T(T+3 S)\left\{-\frac{L_{4}^{(2)}}{9}-\frac{L_{7}^{(3)}}{27} \frac{(S+T)}{2}+\frac{L_{14}^{(4)}}{81}\left(T^{2}-2 S T+5 S^{2}\right)-\right. \\
\left.\quad-\frac{L_{15}^{(4)}}{81}\left(2 T^{2}+6 S^{2}\right)\right\} \\
k_{n}=\left(T^{2}-9 S^{2}\right)\left\{-\frac{L_{4}^{(2)}}{9}+\frac{L_{7}^{(3)}}{27} S+\frac{L_{14}^{(4)}}{81} 2\left(T^{2}+S^{2}\right)-\right. \\
\left.-\frac{L_{15}^{(4)}}{81}\left(2 T^{2}+6 S^{2}\right)\right\}
\end{gathered}
$$

\section{Diseussion}

Starting from a general elastic LGdG free energy (10) and reducing the order parameter space of symmetric and traceless tensors to an order parameter space whose elements satisfy the condition of "hard biaxiality" (16), we derived the elastic theory for biaxial nematic liquid crystals.

The three chiral contributions $k_{l}(\hat{\boldsymbol{l}} \cdot \nabla \times \hat{\boldsymbol{l}}), \quad k_{m}(\hat{\boldsymbol{m}} \cdot \nabla \times \hat{\boldsymbol{m}})$, and $k_{n}(\hat{\boldsymbol{n}} \cdot \nabla \times \hat{\boldsymbol{n}})$ appear naturally in this description and must be included in the generalized free energy for the hard biaxial nematic phase, in accord with the derivation by methods of invariant theory $[8,10,11]$. It would be a highly singular, nongeneric case, if out of the three chiral wavevectors $k_{l} / K_{l 2}, k_{m} / K_{m 2}, k_{n} / K_{n 2}$ one would vanish and the other two would become identical, as requested by Brand and Pleiner [12] for chiral biaxial nematic liquid crystals (this requirement is denied in [7] and [13]).

In the hard biaxial nematic phase, the order parameters $S$ and $T$ both depend on temperature, although the functional relation can only be expressed by numerically determined diagrams, not by an analytic formula. Through $S$ and $T$ also the biaxial elastic constants change with temperature. Measurements of the behaviour of $K_{i j}$ and $k_{l}$ with temperature can in principle be used to estimate the values of the LGdG elastic constants $L_{i}^{(n)}$.

\section{References}

[1] L. Longa, D. Monselesan, and H.-R. Trebin, Liquid Cryst. 2, 769 (1987).

[2] P. G. De Gennes, Mol. Cryst. Liquid. Cryst. 12, 193 (1971).

[3] C. W. Oseen, Trans. Faraday Soc. 29, 883 (1933).

[4] H. Zocher, Trans. Faraday Soc. 29, 345 (1933). 
[5] F. C. Frank, Disc. Faraday Soc. 25, 19 (1958).

[6] E. F. Gramsbergen, L. Longa, and W. H. De Jeo, Physics Rep. 135, 195 (1986).

[7] L. Longa and H.-R. Trebin, Phys. Rev. A 39, 2160 (1989).

[8] H.-R. Trebin, J. Physique 42, 1573 (1981).

[9] H. Brand and H. Pletnke, Phys. Rev. A 24, 2777 (1981); Phys. Rev. A 26, 1783 (1982).

[10] E. Govers and G. Vertogen, Phys. Rev. A 30, 1998 (1984).

[11] E. Govers and G. Vertogen, Phys. Rev. A 31, 1957 (1985).

[12] H. Pleiner and H. R. Brand, Phys. Rev. A 34, 2528 (1986).

[13] E. Govers and G. Vertogen, Phys. Rev. A 34, 2530 (1986). 